\title{
Cytochrome $c$ speeds up caspase cascade activation by blocking 14-3-3e-dependent Apaf-1 inhibition
}

\author{
Carlos A. Elena-Real', Antonio Díaz-Quintana (1]', Katiuska González-Arzola', Adrián Velázquez-Campoy², Mar Orzáez³, \\ Abelardo López-Rivas ${ }^{4}$, Sergio Gil-Caballero, Miguel Á. De la Rosa (i) and Irene Díaz-Moreno
}

\begin{abstract}
Apoptosis is a highly regulated form of programmed cell death, essential to the development and homeostasis of multicellular organisms. Cytochrome $c$ is a central figure in the activation of the apoptotic intrinsic pathway, thereby activating the caspase cascade through its interaction with Apaf-1. Our recent studies have revealed 14-3-3E (a direct inhibitor of Apaf-1) as a cytosolic cytochrome $c$ target. Here we explore the cytochrome c / 14-3-3e interaction and show the ability of cytochrome $c$ to block 14-3-3e-mediated Apaf-1 inhibition, thereby unveiling a novel function for cytochrome $c$ as an indirect activator of caspase-9/3. We have used calorimetry, NMR spectroscopy, site mutagenesis and computational calculations to provide an insight into the structural features of the cytochrome $c / 14-3-3 \varepsilon$ complex. Overall, these findings suggest an additional cytochrome $c$-mediated mechanism to modulate apoptosome formation, shedding light onto the rigorous apoptotic regulation network.
\end{abstract}

\section{Introduction}

Apoptosis, a specific form of programmed cell death (PCD), is an essential process for development and homeostasis of multicellular organisms ${ }^{1}$. There are two main apoptotic pathways in mammalian cells: the extrinsic or death receptor-initiated pathway and the intrinsic or mitochondria-dependent pathway ${ }^{2,3}$. The last is triggered by signals, including DNA damage, oxidative stress, and growth factor deprivation, which converge on the release of pro-apoptotic proteins from the mitochondria into the cytosol; one such protein is cytochrome $c(\mathrm{Cc})$. Under homeostasis, $\mathrm{C} c$ acts as an electron carrier in the mitochondrial respiratory chain ${ }^{4,5}$. Upon its release

\footnotetext{
Correspondence: Miguel A. De la Rosa (marosa@us.es) or Irene Díaz-Moreno (idiazmoreno@us.es)

${ }^{1}$ Instituto de Investigaciones Químicas (IIQ) - Centro de Investigaciones Científicas Isla de la Cartuja (cicCartuja), Universidad de Sevilla - Consejo Superior de Investigaciones Científicas (CSIC), Sevilla, Spain

${ }^{2}$ Institute of Biocomputation and Physics of Complex Systems (BIFI), Joint Unit IQFR-CSIC-BIFI, Universidad de Zaragoza, Zaragoza, Spain

Full list of author information is available at the end of the article Edited by A. Finazzi-Agrò
}

from mitochondria, $\mathrm{C} c$ interacts with WD40 domains of the apoptotic protease activating factor-1 (Apaf-1) in the cytosol, enabling the assembly of the apoptosome ${ }^{6}$. This protein platform activates caspase- 9 and later caspase-3, initiating the caspase cascade, which executes cell death in an orchestrated way ${ }^{7,8}$.

Recent findings suggest $\mathrm{Cc}$ fulfills multiple roles in apoptosis, beyond Apaf-1 activation and apoptosome assembly. Indeed, $\mathrm{C} c$ interacts with various cytosolic and nuclear partners along the onset of $\mathrm{PCD}^{9,10}$. Hence, the full scope of $\mathrm{C} c$ role in apoptosis remains un-elucidated. Recently, our group has reported that $\mathrm{C} c$ inhibits the histone chaperone activity of SET/TAF-I $\beta$ in the nucleus, impairing the formation of core histone-SET/TAF-I $\beta$ complexes under DNA damage ${ }^{11}$. However, the novel functions of cytosolic $\mathrm{C} c$ stay unveiled, despite a complex network of interactions mediated by $\mathrm{C} c$ during apoptosis has been suggested ${ }^{12}$.

Therefore, we focused on the interaction between $\mathrm{Cc}$ and protein 14-3-3e, a novel cytosolic $\mathrm{Cc}$ target under DNA damage ${ }^{10}$. This protein belongs to the 14-3-3 

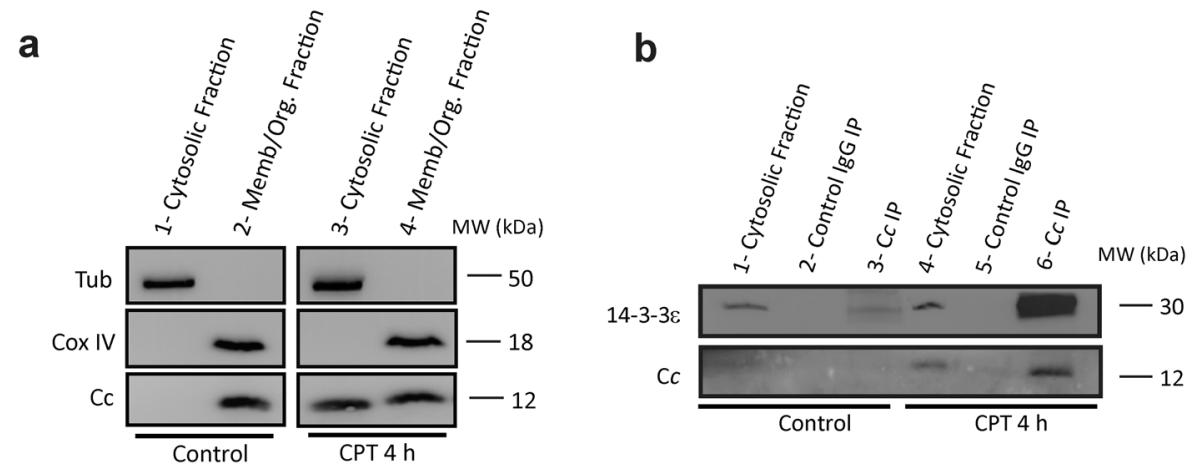

Fig. 1 Cc localization in the cytosol upon cell CPT-treatment. a Subcellular fractioning showing the Cc location upon cell treatment with $20 \mu \mathrm{M}$ CPT for $4 \mathrm{~h}$. Purity of fractions was verified by western blot using anti-a-Tubulin and anti-CoxIV antibodies for detecting cytosolic and membrane proteins, respectively. b Cc-IP of cytosolic fractions from non-treated (lane 3) and CPT-treated (lane 6) along with the detection of 14-3-3ع by western blot (upper). Mouse lgG was used as control (lanes 2 and 5). Verification of immunoprecipitation of Cc upon CPT treatment is shown (lower)

family ${ }^{13,14}$, which comprises seven conserved isoforms $(\beta$, $\gamma, \varepsilon, \eta, \sigma, \tau / \theta$, and $\zeta$ ), arranged as homo- and heterodimers. Each monomer contains nine $\alpha$-helices that form a conserved concave groove, used by 14-3-3 proteins to bind their phosphorylated targets ${ }^{15}$ (Supplementary Figure S1). Furthermore, they are also involved in phosphorylationindependent interactions ${ }^{16-18} \cdot 14-3-3$ proteins participate in several cell processes related to metabolism, signal transduction, cell cycle control, apoptosis, transcription, and stress responses ${ }^{19-24}$.

Among 14-3-3ع functions, its ability to inhibit Apaf-1 stands out because it prevents apoptosome assembly and caspase activation ${ }^{25}$. Such inhibition is enhanced by phosphorylation of Apaf- 1 at Ser268 by the p90kDa ribosomal S6 kinase-1 (Rsk-1) when the mitogenactivated protein kinases (MAPK) cascade is active. Hence, the interaction of $C c$ with $14-3-3 \varepsilon$ could modulate such inhibition somehow.

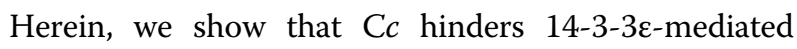
Apaf-1 inhibition. Indeed, our results indicate a competition between $C c$ and $14-3-3 \varepsilon$ for binding to Apaf-1, which enhances caspase activation. Furthermore, this new regulatory mechanism is modulated by phosphorylation of Apaf-1, which enhances its inhibition by 14-3$3 \varepsilon$. We further show that $\mathrm{C} c$ binds to both the $14-3-3 \varepsilon$ concave groove and the convex face, thereby providing a molecular basis for this novel modulation of apoptosome assembly.

\section{Results}

\section{Cc interacts with $14-3-3 \varepsilon$ in the cytosol under apoptotic} conditions

To elucidate the extra-mitochondrial function of $\mathrm{C} c$, we explored the interaction of $\mathrm{C} c$ with 14-3-3e when apoptosis is triggered. To this aim, HeLa cells were treated with the topoisomerase I inhibitor camptothecin (CPT), to induce DNA damage. Then, subcellular fractionation was performed, and $\mathrm{Cc}$ was detected in the cytosol (Fig. 1a, lane 3). However, it remained inside the mitochondria under homeostasis (Fig. 1a, lane 2). The in cell $\mathrm{Cc} / 14-3-3 \varepsilon$ interaction was established as immunoprecipitation (IP) of cytosolic proteins associated with $\mathrm{Cc}$ yielded intrinsic $14-3-3 \varepsilon$ in CPT-treated cells (Fig. 1b, lane 6). To further confirm the IP specificity, untreated and CPT-treated cytosolic lysates were probed with a 14-3-3e antibody (Fig. 1b, lanes 1 and 4, respectively). Negative controls using IgG (Fig. 1b, lanes 2 and 5) did not display any band. Immunoblotting against the anti-Cc antibody confirmed Cc IP (Fig. 1b, lane 6).

\section{Cc blocks 14-3-3ع-mediated caspase inhibition}

Following its release into the cytosol, $\mathrm{C} c$ targets Apaf-1 to assemble the apoptosome ${ }^{6}$. As 14-3-3e binds Apaf- 1 to prevent caspase activation ${ }^{25}$, we investigated whether $\mathrm{Cc}$ modulates Apaf-1 inhibition by 14-3-3ع.

First, we checked the ability of $\mathrm{C} c$ to activate caspase- 3 in HEK293T cytosolic cell extracts. Caspase-3 activity was monitored upon $\mathrm{C} c$ addition (Fig. 2a, white columns), resulting in a substantial increase of such activity, as the hemeprotein triggered the apoptosome formation and, subsequently, caspase- $9,-3$ activation.

MAPK proteins modulate Apaf- 1 activity, leading the activation of Rsk-1, which phosphorylates Apaf-1 at Ser268, favoring the interaction between Apaf-1 and 14-3$3 \varepsilon$, thereby increasing Apaf-1 inhibition ${ }^{25}$. Therefore, we tested the effect of phorbol 12-myristate 13-acetate (PMA), which triggers the MAPK cascade, on the caspase3 activity. Apaf-1 phosphorylation under PMA treatment was confirmed by Phos-tag ${ }^{\text {TM }}$ SDS PAGE (Supplementary Figure S2). PMA-treated cells extracts showed a lower caspase- 3 activity than untreated extracts in the presence of Cc (Fig. 2a, gray columns). This is consistent with Apaf1 phosphorylation at Ser268, enhancing its inhibition by $14-3-3 \varepsilon^{25}$. 
a

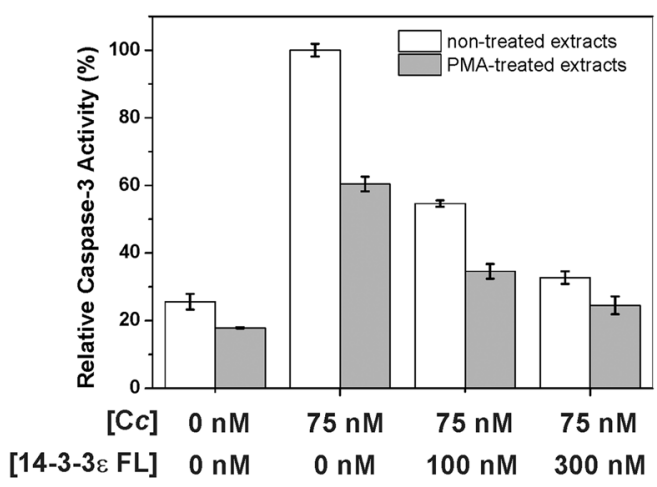

C

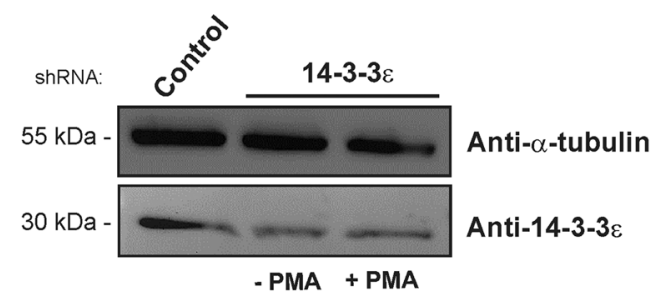

b

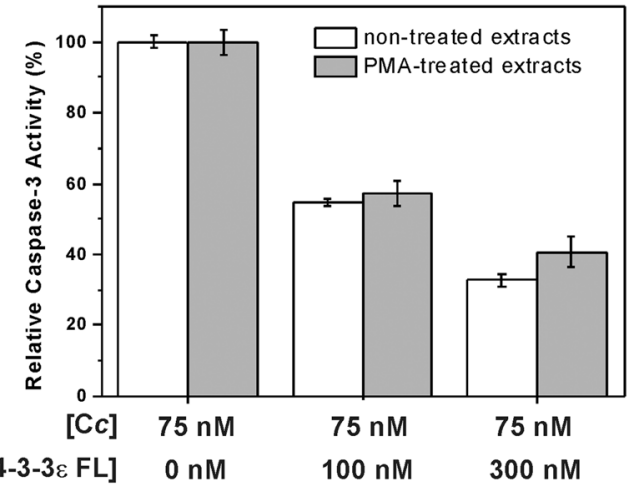

d

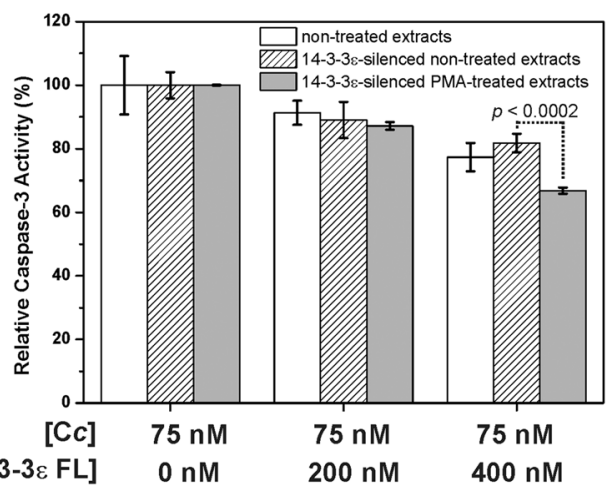

Fig. 2 14-3-3 $\varepsilon$ FL inhibits caspase-3 activity in HEK293T cytosolic cell extracts. a Relative caspase-3 activity in non-treated (white columns) and PMA-treated (gray columns) HEK293T cytosolic cell extracts were measured upon addition of recombinant Cc and increasing concentrations of 14-3$3 \varepsilon$ FL. b Normalized data shown in a. c HEK293T cells were transfected with empty pSUPER (Control) or with pSUPER-shRNA against 14-3-3E and

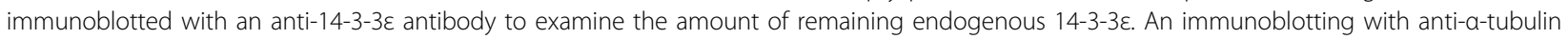
was used as loading control. $\mathbf{d}$ Normalized caspase-3 activity in 14-3-3e-silenced non-treated and PMA-treated cell extracts upon addition of recombinant $14-3-3 \varepsilon$ FL. Normalized caspase-3 activity of non-14-3-3e-silenced, non-treated cell extracts is included as a control

Then, we tested the ability of recombinant $14-3-3 \varepsilon$ (herein named as $14-3-3 \varepsilon$ full length, $14-3-3 \varepsilon$ FL) to inhibit caspase- 3 activity in non-treated and PMA-treated cell extracts. Addition of 14-3-3e FL made extracts to exhibit significantly lower $\mathrm{C} c$-mediated caspase- 3 activity values (Fig. 2a). These were normalized to identify the particular effect of added 14-3-3e FL but, unexpectedly, the normalization evinced that the inhibition exerted by 14-3-3 $\varepsilon$ FL was similar in non-treated and PMA-treated extracts (Fig. 2b). One of the plausible explanations for this observation is endogenous 14-3-3 $\varepsilon$ already abolished the caspase- 3 activity by preferentially binding to phosphorylated Apaf-1, thus preventing any additional effect of exogenous 14-3-3e FL. Additional caspase-3 assays were carried out using extracts from 14-3-3ع knockdown HEK293T cells (Fig. 2c), with the caspase-3 activity in PMA-treated extracts resulting more sensitive than nontreated extracts to the addition of 14-3-3e FL (Fig. 2d).

In summary, these findings demonstrate how $14-3-3 \varepsilon$ FL inhibits caspase activity, and how this inhibitory mechanism can be modulated by Apaf-1 phosphorylation. Nevertheless, the inhibitory effect of $14-3-3 \varepsilon$ FL on caspase- 3 could be caused by either a direct interaction with Apaf-1 or by hindering the complex between the latter and $C c$. Caspase- 9 can indeed be phosphorylated by the ERK MAPK pathway, with the resulting modulation of its function and consequently, of caspase-3 activation ${ }^{26}$.

To discard the direct interaction between $\mathrm{C} c$ and Apaf1, as well as the ERK-mediated inhibition of caspase-9, we performed caspase- 9 activity assays using a recombinant, truncated form of Apaf-1 lacking the $\mathrm{C} c$-binding WD40 domains $\left(\text { Apaf- } 1_{\triangle W D 40}\right)^{27}$. Apaf- $1_{\triangle W D 40}$ activates caspase9 in a constitutive manner in the absence of $\mathrm{Cc}$ (Fig. 3). The inability of $\mathrm{C} c$ to alter the Apaf- $1_{\triangle W D 40}$-mediated caspase- 9 activity was likewise determined (Supplementary Figure S3). Then, the addition of 14-3-3e FL inhibited caspase- 9 activity strongly, due to the binding of Apaf$1_{\Delta \mathrm{WD} 40}$ to $14-3-3 \varepsilon$ FL (Fig. 3 ). However, the sequentially titration with $\mathrm{C} c$ restored caspase- 9 activity, indicating a competition between $\mathrm{C} c$ and Apaf-1 $1_{\Delta \mathrm{WD} 40}$ for the binding 


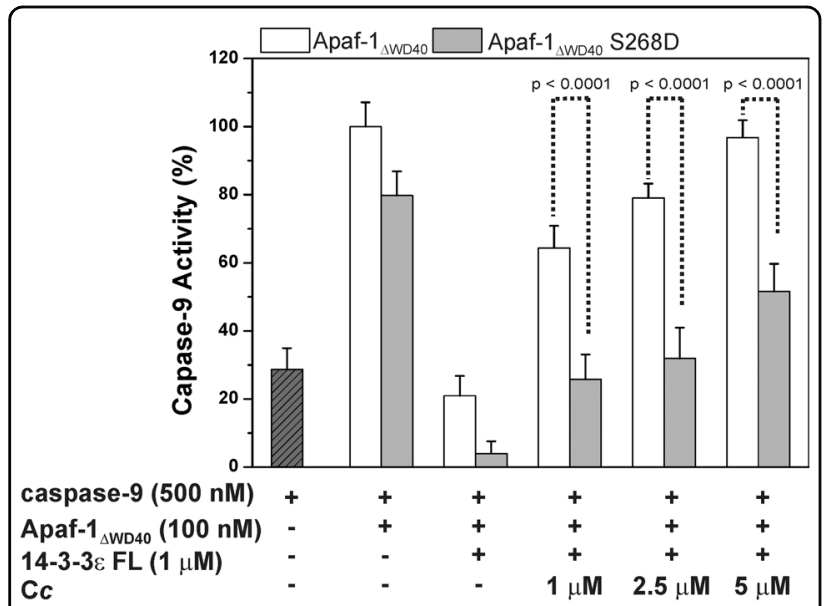

Fig. 3 Cc impairs 14-3-3 $\varepsilon$ FL-mediated caspase-9 inhibition. Cc and $14-3-3 \varepsilon$ FL were added to caspase- 9 and different Apaf- ${ }_{\triangle W D}{ }_{W} 40$ mutants

site of 14-3-3e FL, impairing Apaf-1 inhibition. Nuclear magnetic resonance (NMR) experiments corroborated such a competition. Specifically, the $\varepsilon$-methyl group signal of Met80 in reduced $\mathrm{C} c$ substantially broadened upon binding of the hemeprotein to 14-3-3e (Supplementary Figure S3e). Further addition of Apaf- $1_{\triangle W D 40}$ yielded partial recover of the signal intensity, indicating that Apaf- $1_{\triangle W D 40}$ competes with $C c$ for the 14-3-3e FL binding site. The inability of Apaf- $1_{\triangle \mathrm{WD} 40}$ to bind $C c$ was corroborated (Supplementary Figure S3f).

As phosphorylation of Apaf-1 at Ser268 enhances its interaction with $14-3-3 \varepsilon \mathrm{FL}$, we tested the ability of $\mathrm{C} c$ to modulate the 14-3-3e FL / Apaf- $1_{\Delta W D 40}$ complex upon phosphorylation. To this end, we designed the recombinant Apaf- $1_{\triangle W D 40}$ S268D mutant, which mimics Ser268 phosphorylation. As a control of unspecific effects caused by mutations at such position, the Apaf- $1_{\triangle W D 40}$ S268A variant was also tested. Notably, the ability of phosphomimetic Apaf- $1_{\triangle \mathrm{WD} 40}$ S268D mutant to trigger caspase-9 activity was similar to that exhibited by Apaf- $1_{\triangle W D} 40$. Nevertheless, the Apaf- $1_{\triangle W D 40}$ S268D-mediated caspase9 activity was slightly more sensitive to $14-3-3 \varepsilon$ (Fig. 3, gray columns). Indeed, the recovery of enzymatic activity by $\mathrm{C} c$ was substantially lower than that measured using Apaf- $1_{\triangle W D 40}$ (Fig. 3, gray columns). Such a finding indicates a larger affinity of the phosphorylated Apaf- 1 species toward $14-3-3 \varepsilon$ that displaces $\mathrm{Cc}^{25}$. On the other hand, the caspase- 9 activation in the presence of the S268A mutant behaved in a way similar to that in the presence of Apaf- $1_{\triangle W D 40}$ (Supplementary Figure S3b). Hence, the negative charge at position 268 in Apaf-1 is responsible for the increase in affinity of Apaf-1 toward 14-3-3e FL.

Our results thus support a model in which $\mathrm{C} c$ activates Apaf-1 while blocking the inhibition exerted by $14-3-3 \varepsilon$ on the apoptotic factor (Fig. 4).

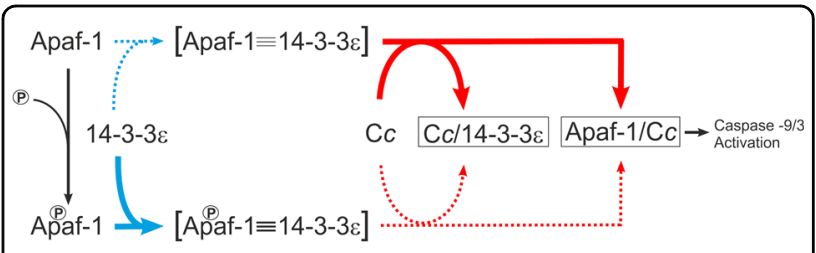

Fig. 4 Model of Apaf-1 regulation by $C c$ and 14-3-3ع. Model of Apaf-1 regulation by $\mathrm{Cc}$ and $14-3-3 \varepsilon$. During homeostasis, Apaf-1 is available to interact with $14-3-3 \varepsilon^{25}$. Upon its release into the cytosol, Cc competes with Apaf-1 for the binding to $14-3-3 \varepsilon$, impairing the Apaf-1 / 14-3-3E complex. Thus, Apaf-1 is available to interact with CC, thereby activating the caspases cascade ${ }^{6}$. Upon Apaf-1 phosphorylation, the equilibrium of the reaction is strongly shifted toward the Apaf-1 / 14-3-3E complex formation. This would hinder the competition performed by $C c$, thereby partly impairing the activation of the caspases

\section{Cc recognizes two different binding sites of $14-3-3 \varepsilon$ full length}

The dimerization state of $14-3-3 \varepsilon$ FL was confirmed by analytic ultracentrifugation combined with dynamic light scattering (Supplementary Figure S4a-c). Sedimentation velocity experiments confirmed the dimeric state of 14-3$3 \varepsilon \mathrm{FL}$ with a molecular mass of $66.4 \mathrm{kDa}$ (Supplementary Figure S4b). Dynamic light scattering measurements corroborated that $14-3-3 \varepsilon$ FL behaves as a dimer, with a particle size of $9 \pm 2 \mathrm{~nm}$ (Supplementary Figure S4c).

Isothermal titration calorimetry (ITC) experiments in Supplementary Figure S5 revealed that $\mathrm{C} c$ binds to 14-3$3 \varepsilon$ FL homodimer with a 2:1 stoichiometry and a dissociation constant $\left(K_{\mathrm{D}}\right)$ of $2.3 \mu \mathrm{M}$ (Supplementary Table S1). To reveal $14-3-3 \varepsilon$ residues implicated in the interaction with Cc, 14-3-3e FL D21K, K50E, S59E, E92K, D99K, and S187D mutants were designed. Residues were selected according to their role in the interactions of 14-33 isoforms with their respective partners ${ }^{28-30}$ and the impact of each mutation in the electrostatic surface potential of the protein. The dimeric state of these mutants was confirmed by analytic ultracentrifugation and dynamic light scattering (Supplementary Figure S4c and d). According to ITC analysis (Supplementary Table S1 and Figure S5), the six mutants bound to $\mathrm{C} c$ with slightly larger $K_{\mathrm{D}}$ values than 14-3-3e FL WT. Further, the complexes involving 14-3-3e FL D21K, K50E, S59E, or E92K showed a 1:1 stoichiometry instead of the 2:1 found for the WT species (Supplementary Table S1), suggesting that one of the two Cc-binding sites of 14-3-3e FL has been compromised in these mutants. Therefore, Asp21, Lys50, Ser59, and Glu92 residues may be essential in Cc recognition.

We also performed NMR experiments to further characterize the interaction between reduced $\mathrm{C} c$ and 14-3-3e FL. We followed Cc signals upon titration of $14-3-3 \varepsilon$ FL, which caused an overall broadening of $\mathrm{C} c$ resonances 


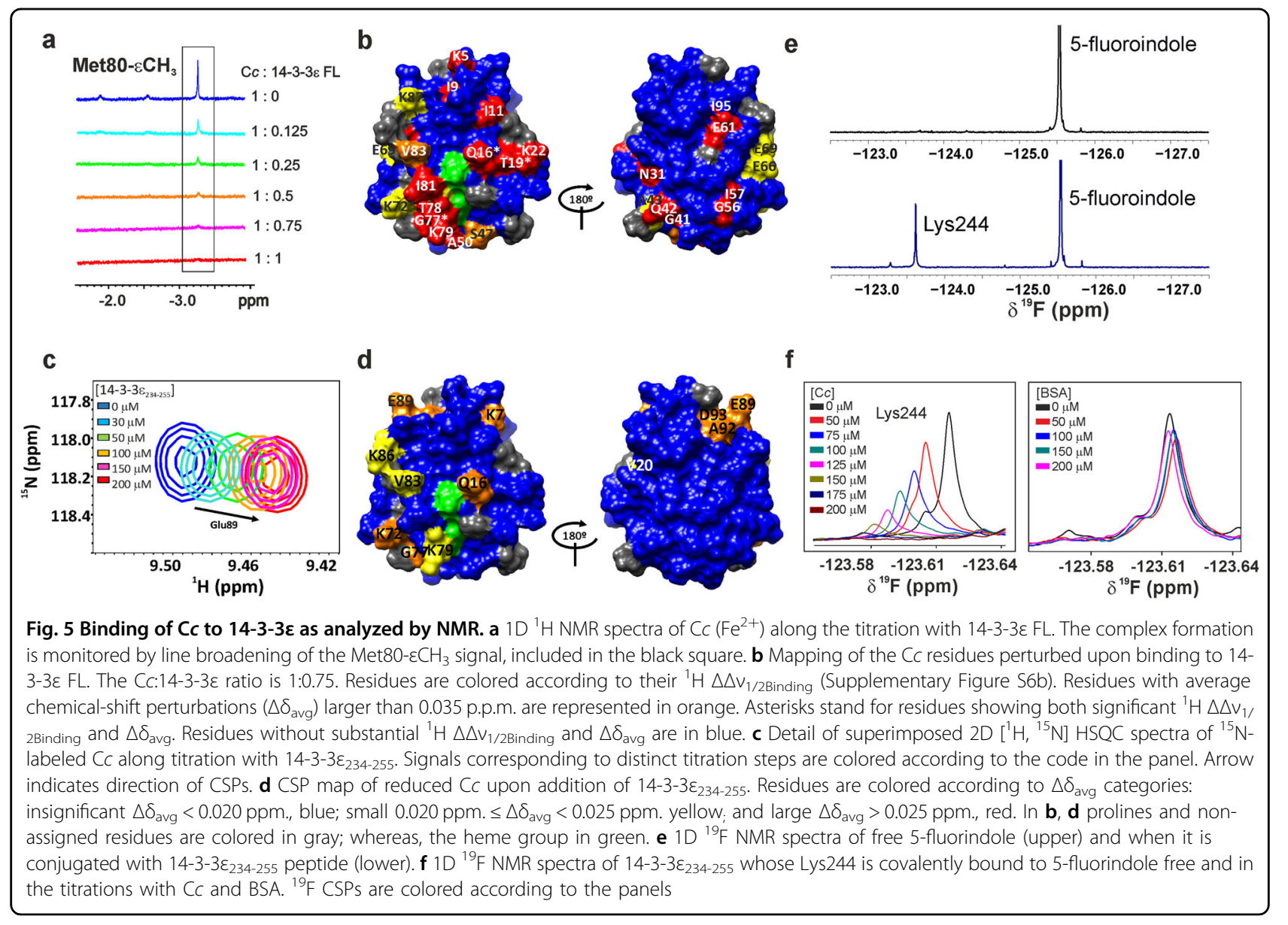

because Cc / 14-3-3e FL complex formation (Fig. 5a and Supplementary Figure S6a, left). Specifically, the $\varepsilon$-methyl group signal of the Met80 $\mathrm{Cc}$ residue was largely broadened at 1:0.5 Cc:14-3-3e FL ratio (Fig. 5a), in agreement with the stoichiometry inferred from ITC data. Moreover, specific $\mathrm{C} c$ backbone amide groups underwent singularly large changes in their ${ }^{1} \mathrm{H}$ line widths $\left({ }^{1} \mathrm{H} \Delta \Delta v_{1 / 2}\right.$ Binding $)$ upon binding with 14-3-3e FL (Supplementary Figure S6b). These resonances were expected to be at or in the proximity of the area of $\mathrm{C} c$ involved in the recognition of $14-3-3 \varepsilon$ FL. Moreover, several signals displayed substantial chemical-shift perturbations (CSPs) in the presence of 14-3-3e FL (Supplementary Figure S6a, right). Specifically, Gln16, Thr19, Ser47, Lys72, Gly77 and Val83 signals showed significant CSPs $\left(\Delta \delta_{\text {avg }}>0.035\right.$ $\mathrm{ppm}$.). Interestingly, the signal corresponding to Val83 (Supplementary Figure S6a, right) seems to be in conformational exchange in the free $\mathrm{C} c$, fixing its conformation upon the interaction with 14-3-3e. These effects are consistent with an intermediate exchange rate at the NMR time scale, in agreement with the transient nature of this complex and the $K_{\mathrm{D}}$ values in the $\mu \mathrm{M}$ range.
Both broadening and CSPs maps on Cc surface (Fig. 5b) indicate that $\mathrm{C} c$ mainly recognizes $14-3-3 \varepsilon$ FL by a surface patch surrounding the heme cleft, as it does in its homeostatic interactions in the electron transport chain $^{31-33}$.

\section{The C-terminal tails of $14-3-3 \varepsilon$ are important in the interaction with $\mathrm{Cc}$}

To decipher the role of the unstructured C-terminal tail of $14-3-3 \varepsilon \mathrm{FL}$ during $\mathrm{Cc}$ recognition, the interaction between $\mathrm{C} c$ and the $14-3-3 \varepsilon$ core protein, lacking Cterminal tails, was measured by ITC. The recorded thermograms showed very similar thermodynamic parameters for both $14-3-3 \varepsilon$ FL and $14-3-3 \varepsilon$ core proteins interacting with $\mathrm{C} c$, although a slight increase in the $K_{\mathrm{D}}$ value was observed in the interaction between $\mathrm{C} c$ and the 14-3-3e core (Supplementary Table S1). This suggests that the Cterminal tails of $14-3-3 \varepsilon$ may participate in the interaction with $\mathrm{C} c$, despite being unessential.

To confirm that the C-terminal tails of 14-3-3e (named $\left.14-3-3 \varepsilon_{234-255}\right)$ are involved in the interaction with $\mathrm{C} c, 2 \mathrm{D}$ $\left[{ }^{1} \mathrm{H},{ }^{15} \mathrm{~N}\right]$ HSQC were recorded along a titration of 14-3$3 \varepsilon_{234-255}$ peptide onto ${ }^{15} \mathrm{~N}$-labeled reduced $\mathrm{Cc}$ 


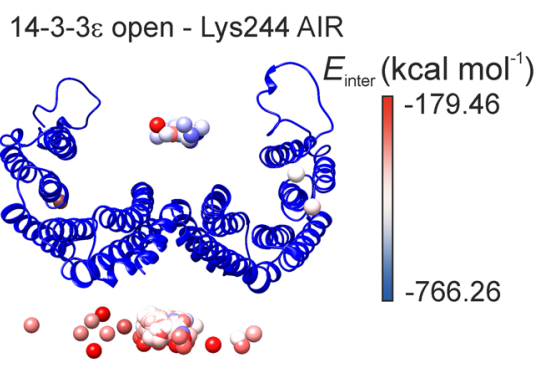

14-3-3ع open + Lys244 AIR

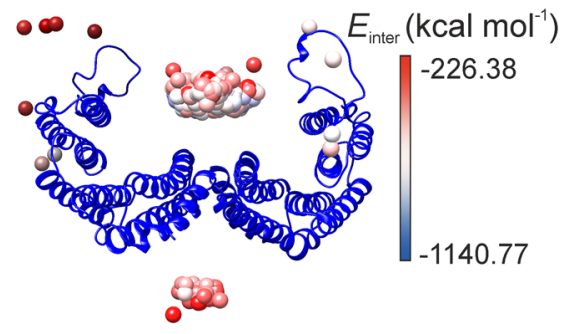

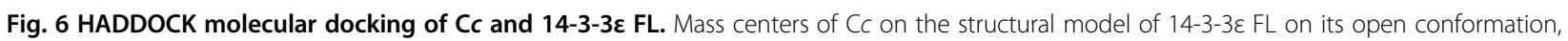
including (right) or not (left) Lys 244 as an active residue (AIR). Mass centers of Cc are colored as a function of intermolecular energy ( $E_{\text {inter }}$ ) according to the scales represented in their corresponding panels

(Supplementary Figure S6c). Such titration resulted in significant CSPs of the $\mathrm{Cc}$ amide signals (Fig. 5c). The resulting interaction surface (Fig. $5 \mathrm{~d}$ ) indicates that 14-3$3 \varepsilon_{234-255}$ binds to the rim of the heme groove of $\mathrm{C} c$, as with 14-3-3e FL protein. However, unlike the 14-3-3e FL interaction, most of the $\mathrm{Cc}$ resonances were in fast exchange on the NMR time scale upon binding to the 143-3 $\varepsilon_{234-255}$ peptide. ${ }^{1} \mathrm{H} \Delta \delta_{\text {bind }}$ was fitted using the best 1:1 binding model, obtaining a $K_{\mathrm{D}}$ of $49 \mu \mathrm{M}$ (Supplementary Figure S6d). Moreover, ITC experiments in which the 143-3 $\varepsilon_{234-255}$ peptide was titrated onto $C c$ yielded a $K_{\mathrm{D}}$ value of $16 \mu \mathrm{M}$ (Supplementary Figure S5 and Table S1). Both NMR and ITC $K_{\mathrm{D}}$ values are marginally higher than the value obtained for the interaction between $14-3-3 \varepsilon \mathrm{FL}$ and Cc. Furthermore, the ITC analysis also confirmed a 1:1 stoichiometry.

Then, to easily determine a residue which could be included in the latter docking calculations, the only one positively charged residue (Lys244) of $14-3-3 \varepsilon_{234-255}$ peptide was tagged with 5 -fluorindole molecule through a conjugation reaction. To prevent the reaction of 5fluorindole with the $\mathrm{N}$-terminal $-\mathrm{NH}_{3}{ }^{+}$group, the peptide was modified by acetylation. To confirm that the acetylation in the $\mathrm{N}$-terminal residue did not drastically modify the interaction with $\mathrm{C} c$, ITC experiments were performed with the acetylated 14-3-3 $\varepsilon_{234-255}$. Both the non-acetylated and acetylated peptides interacted with $\mathrm{Cc}$ in a similar way, despite the $K_{\mathrm{D}}$ value showed a slight increase when the peptide was acetylated (Supplementary Table S1).

Proper folding of $14-3-3 \varepsilon_{234-255}$ before and after the conjugation reaction was unambiguously assessed by $1 \mathrm{D}$ ${ }^{1}$ H NMR spectra (Supplementary Figure S6e). Second, 1D ${ }^{19} \mathrm{~F}$ NMR spectra of both free 5-fluorindole and fluorinated $14-3-3 \varepsilon_{234-255}$ were registered. The spectrum of fluorinated $14-3-3 \varepsilon_{234-255}$ peptide showed a signal at $-125.53 \mathrm{ppm}$. corresponding to free 5 -fluorindole, and another at $-123.62 \mathrm{ppm}$. that was assigned to 5fluorindole conjugated to the epsilon $-\mathrm{NH}_{3}{ }^{+}$group of Lys244 (Fig. 5e). The addition of increasing concentration of $\mathrm{Cc}$ resulted in significant CSPs of this second signal (Fig. 5f, left panel). Notably, the ${ }^{19}$ F NMR signal also experienced a significant broadening consistent with the larger correlation time of the peptide bound to $\mathrm{C} c$.

As a negative control, bovine serum albumin (BSA) protein was titrated into fluorinated $14-3-3 \varepsilon_{234-255}$ peptide, with the negligible resulting ${ }^{19} \mathrm{~F}$ CSPs, suggesting that BSA cannot significantly bind to the peptide. (Fig. 5f, right panel). These results agree with Lys244 of 14-3-3e FL taking part in Cc recognition. Therefore, Lys 244 was included as an active residue in further docking calculations (see below).

\section{Structural model of the interaction between $C c$ and 14-3- $3 \varepsilon$ full length}

To further understand the molecular features of the interaction between $\mathrm{C} c$ and 14-3-3e FL, we performed restrain-driven docking computations using HADDOCK software $^{34}$. For these computations, we used the structural model of $14-3-3 \varepsilon$ FL in its open conformation inferred from MD as the input structure (Supplementary Information and Figure S7), to allow the entrance of $\mathrm{Cc}$ into the concave groove of the 14-3-3e dimer. Line widths and CSPs obtained from NMR analysis of the Cc / 14-3-3e FL complex were used as $\mathrm{C} c$ ambiguous interaction restrains (AIRs). The 14-3-3e FL residues that resulted in the impairment of one of the $\mathrm{C} c$-binding sites by ITC were used as $14-3-3 \varepsilon$ AIRs. The 500 solutions obtained in the computation showed two main Cc-binding sites on 14-3$3 \varepsilon$ FL dimer, in agreement with the stoichiometry inferred from ITC data. These solutions located $\mathrm{C} c$ in both the concave and convex faces of $14-3-3 \varepsilon$; these interacting regions will be referred hereafter to as the concave and convex binding sites, respectively. The convex site resulted more populated, although some of the lowest energy solutions locate within the concave site (Fig. 6, left panel). Statistical classification of solutions yielded three clusters in the convex site of $14-3-3 \varepsilon$ and only one in the concave site (Supplementary Figure S8a). Further analyses indicate that electrostatics is the major contributing factor to 
binding energy in the three clusters (Supplementary Table S2). However, the concave cluster was more energetically favored than the convex clusters, mainly due to a lower electrostatic energy $\left(E_{\text {elec }}\right)$. Nevertheless, convex clusters presented lower AIRs violations than the concave cluster.

Furthermore, to study the role of the $14-3-3 \varepsilon$ Cterminal tails in the interaction, a docking computation was also performed including Lys244 (located in the Cterminal tail), as an active residue. This computation showed an increase in the number of solutions locating $\mathrm{C} c$ in the concave binding site of 14-3-3 $\varepsilon$ (Fig. 6, right panel), suggesting that the $\mathrm{C}$-terminal tails contribute to the entrance of $\mathrm{Cc}$ into the concave groove of 14-3-3e. Clustering analysis exhibited three clusters of solutions presenting $C c$ bound to the concave site of $14-3-3 \varepsilon$, and only one displaying $\mathrm{Cc}$ bound to the convex site (Supplementary Figure S8b). Again, electrostatic interactions constituted the major contribution in all clusters, concave site clusters displaying lower energies because of their $\mathrm{E}_{\text {elec }}$ term (Supplementary Table S2).

Structural models of Cc / 14-3-3e FL complex were obtained for computations excluding and including (Supplementary Figure S8c and d, respectively) Lys244 as an active residue of 14-3-3e. For this, the best two complexes with minimal $E_{\text {inter }}$ at each binding site of 14-3-3e were selected from each computation. Both structural models present a $\mathrm{C} c$ molecule located in the concave groove of $14-3-3 \varepsilon$, contacting at least one of the Cterminal tails of $14-3-3 \varepsilon$ dimer. A second $\mathrm{C} c$ molecule is placed at the convex side of $14-3-3 \varepsilon$, specifically at the rim of the dimerization surface thereby binding both 14-3-3 monomers. In addition, the two $\mathrm{Cc}$ molecules from both computations present their heme clefts oriented to 14-3$3 \varepsilon$, according to NMR data.

\section{Discussion}

Due to the apoptosis significance in multicellular organisms, its regulation relies on a subtle balance between inhibition and activation pathways. In fact, signaling cascades convert continuous stimuli in an all-ornone response to avert physiological fluctuations to trigger a response accidentally ${ }^{35}$. Feedbacks are needed to lead response ultra-sensitivity: a large stimulus is required to trigger a response, which changes dramatically within a narrow range of stimulus intensity. During homeostasis, apoptotic factors remain inhibited, either blocked by other proteins or sequestered in a particular cell compartment. Along apoptosis, several apoptotic factors are released from the mitochondria to the cytosol. Among these factors is $\mathrm{Cc}$, which promotes the apoptosome assembly through its association with Apaf-1, thereby initiating the caspase cascade ${ }^{6}$. On the other hand, prosurvival pathways, as the inhibition of Apaf-1 by 14-3-
$3 \varepsilon^{25}$, reduce the sensitivity to the apoptosis signaling network.

Therefore, interactions between pro-apoptotic and prosurvival proteins, such as that between $C c$ and 14-3-3e, are of particular relevance as they provide additional regulation loops. In this study, we have shown that $\mathrm{Cc}$ interacts with $14-3-3 \varepsilon$ in the cytosol under apoptotic conditions, preventing the binding of $14-3-3 \varepsilon$ to a specific site in Apaf-1. Hence, this interaction could be capable of regulating caspase activity. Indeed, $\mathrm{C} c$ enhanced caspase activation in presence of $14-3-3 \varepsilon$ inhibitor and a truncated, constitutive form of Apaf- 1 unable to bind the hemeprotein. Thus, $C c$ plays a double role in the activation of caspase cascade directly by interacting with Apaf1 , and indirectly by blocking $14-3-3 \varepsilon$ thereby releasing Apaf-1. This double role enables $\mathrm{C} c$ to inhibit pro-survival pathways while apoptotic routes have been triggered (Fig. 4).

Further phenomena modulate the cell fate signaling, including protein phosphorylation events, as those which regulate $\mathrm{C} c$ functions, including its ability to activate caspases $^{36-40}$. In addition, such a phosphorylation enhances the inhibition of Apaf- 1 by $14-3-3 \varepsilon^{25}$, making caspase activation less sensitive to $\mathrm{C} c$. In fact, activation of MAPK cascade favors the Apaf-1 inhibition by 14-3-3ع. Similarly, the phosphomimetic Apaf-1 mutant is more susceptible to 14-3-3e-dependent inhibition, which consequently becomes less sensitive to the presence of $\mathrm{C} c$. Hence, this phosphorylation of Ser268 in Apaf-1 may attenuate the effect of $\mathrm{C} c$ release when caspase activation is undesirable (Fig. 4).

$\mathrm{Cc}$ does lack the consensus sequence which 14-3-3e usually recognizes in its various targets. Our biophysical analysis has revealed that two molecules of $\mathrm{C} c$ can interact with $14-3-3 \varepsilon$ at two different sites: the groove of $14-3-3 \varepsilon$ where its targets bind, and the convex side of the dimer. Additionally, the data herein suggest that flexible Cterminal $14-3-3 \varepsilon$ may recognize $C c$ without being essential for the binding. These tails are flexible and mostly unstructured, although MD trajectories indicate the presence of residual helical elements. Moreover, NMR data show that $C c$ recognizes $14-3-3 \varepsilon$ by residues surrounding the heme cleft, as reported for other interactions involving the hemeprotein ${ }^{31-33}$.

In summary, $C c$ is revealed as a double Apaf- 1 activator via two mechanisms: by directly binding to the apoptosis factor and impairing its inhibition by 14-3-3e. Indeed, such a cooperative behavior of $\mathrm{C} c$, triggering effector pathways while modulating feedbacks loops make cell response ultrasensitive to its release. The analysis of the various additional interactions of $\mathrm{Cc}$ during the onset of programmed cell death recently unveiled may confirm if this conclusion can be extended as a general mechanism by which $C c$ triggers apoptosis ${ }^{9-12,41}$. 


\section{Materials and methods Cell cultures}

HeLa and Human Embryonic Kidney 293T (HEK293T) cells were cultured in Dulbecco's modified Eagle's medium (DMEM), supplemented with 10\% heat-inactivated fetal bovine serum (FBS), $2 \mathrm{mM}$ L-glutamine, $100 \mathrm{U} / \mathrm{mL}$ penicillin, $100 \mu \mathrm{g} / \mathrm{mL}$ streptomycin, and maintained at 37 ${ }^{\circ} \mathrm{C}$ in a humidified $5 \% \mathrm{CO}_{2}$ atmosphere.

For caspase- 3 assays, $4.5 \times 10^{6}$ HEK293T cells were cultured in $140 \mathrm{~mm}$ Petri dishes in DMEM containing FBS until 70\% confluence was reached, and cells were then kept in DMEM without FBS for $4 \mathrm{~h}$. In some experiments (indicated in the main text) cells were treated with $50 \mathrm{ng} / \mathrm{mL}$ phorbol-12-myristate-13-acetate (PMA, Sigma-Aldrich) or treated with the same volume of DMSO for $120 \mathrm{~min}$.

\section{shRNA knockdown}

In order to knockdown 14-3-3e expression in HEK293T cells, a construct containing the shRNA sequence (5'-GATCCCCTGTTCAATACTGCTGATTATTCTCCTTCAAGAGAGGAGAATAATCAGCAGTATTGAACATTTTTA-3') was cloned into a pSUPER vector using BgIII-HindIII restriction sites. An empty pSUPER vector was used as a control. Cells were transfected with the corresponding PSUPER plasmid by calcium phosphate method, as described elsewhere $^{42}$. Transfected cells were selected by adding puromycin $(1.5 \mu \mathrm{g} / \mathrm{mL})$ to the culture medium. Four days after transfection, cells were collected and cytosolic extracts were obtained.

\section{Subcellular fractionation and extract preparation}

For immunoprecipitation assays, $6 \times 10^{6} \mathrm{HeLa}$ cells were seeded in $140 \mathrm{~mm}$ Petri dishes and treated with an apoptosis inducing agent $(20 \mu \mathrm{M}$ camptothecin, CPT) for $4 \mathrm{~h}$. HeLa cells were fractionated into cytosolic, membrane/organelles and nuclear fractions using a ProteoExtract Subcellular Proteome Extraction Kit (Calbiochem) following the manufacturer's indications. The purity of subcellular fractions obtained was verified by western blot analysis, using anti- $\alpha$-tubulin and anti-COX IV for detecting cytosol and mitochondria, respectively.

For caspase-3 activity assays, cytosolic extracts were obtained from HEK293T cells as previously described with briefly modifications ${ }^{43}$. Briefly, cells were trypsinized and collected by centrifugation ( $5 \mathrm{~min}$ at $1500 \times \mathrm{g}$ ) and washed twice with phosphate buffer saline (PBS) and once with Cellular Extract Buffer (CEB, $20 \mathrm{mM}$ HEPES at $\mathrm{pH}$ 7.5, $10 \mathrm{mM} \mathrm{KCl,} 1.5 \mathrm{mM} \mathrm{MgCl} 2,1 \mathrm{mM}$ Na-EDTA, $1 \mathrm{mM}$ EGTA, $1 \mathrm{mM}$ dithiothreitol [DTT], $100 \mu \mathrm{M}$ PMSF). Two volumes of $\mathrm{CEB}$ were added to cells, and they were incubated for $15 \mathrm{~min}$ on ice, allowing the hypotonic swell of cells. The cells were then disrupted by $20-25$ strokes with a Douncer homogenizer and lysates were centrifuged at $15,000 \times g$ for $15 \mathrm{~min}$ at $4{ }^{\circ} \mathrm{C}$ to remove organelles.

\section{Immunoprecipitation}

Cytosolic fractions from subcellular fractionation of control and CPT-treated HeLa cells were used to immunoprecipitate $\mathrm{Cc}$. Quantity of $300 \mu \mathrm{g}$ of cytosolic samples were incubated with $50 \mu \mathrm{L}$ Sepharose 6B (Sigma-Aldrich) for $2 \mathrm{~h}$ at $4{ }^{\circ} \mathrm{C}$ under agitation as a pre-clearing step to reduce non-specific binding. Samples were centrifuged and $20 \mu \mathrm{L}$ of rabbit anti-human cytochrome $c(\mathrm{Cc})$ were added to supernatants and incubated overnight at $4{ }^{\circ} \mathrm{C}$ under rotation. As a negative control, $300 \mu \mathrm{g}$ of cytosolic lysates were incubated with $1 \mu \mathrm{g}$ of anti-mouse IgG under the same conditions. Afterwards, $50 \mu \mathrm{L}$ Protein A Sepharose (GE Healthcare) was added to each lysate for 4 $\mathrm{h}$ at $4{ }^{\circ} \mathrm{C}$ under rotation. The protein-Sepharose complexes were washed extensively, collected by centrifugation and boiled in freshly prepared reducing loading buffer. Controls including $30 \mu \mathrm{g}$ of cytosolic lysates were run concurrently with the immunoprecipitated (IP) samples in the western blot assays.

Apaf-1 immunoprecipitation was performed from total non-treated and PMA-treated lysates. Apaf-1 IP samples were analyzed by western blot using a Phos-tag ${ }^{\text {TM }}$ SDS PAGE (Wako) - which reduce the electrophoretic mobility of phosphorylated proteins.

\section{Antibodies}

Rabbit anti-human $\mathrm{Cc}$ serum was obtained after immunizing male rabbits with full length recombinant $\mathrm{Cc}$ suspended in a $0.85 \%$ solution of $\mathrm{NaCl}(20 \mathrm{mg} / \mathrm{mL})$. The suspension was then incorporated into an equal amount of complete (Freund) adjuvant obtained from Difco (BD Biosciences, US). Mouse monoclonal anti- $\alpha$-Tubulin (catalogue number T8328), as well as secondary horseradish peroxidase (HRP)-conjugated anti-mouse IgG (catalogue number A4416), rabbit polyclonal anti-14-3-3e (catalogue number SAB4503100), and anti-rabbit IgG (catalogue number A0545) were obtained from SigmaAldrich. Rabbit polyclonal antibody to cytochrome $c$ oxidase subunit IV (COX IV) was from Abcam (catalogue number ab16056). Mouse monoclonal anti-Apaf-1 (catalogue number sc-135836) was obtained from Santa Cruz Biotechnology, INC. Mouse monoclonal antiphosphoserine (Millipore clone A4) was obtained from Merck.

\section{Western blot analysis}

For the immunoblot detection of $\mathrm{C} c, \alpha$-Tubulin, COX IV, and $14-3-3 \varepsilon$ in the subcellular fractions or IP samples, protein content was measured using the $\mathrm{DC}^{\mathrm{TM}}$ protein assay (Bio-Rad Laboratories, US). Proteins were resolved by sodium dodecyl sulphate-polyacrylamide gel 
electrophoresis (SDS-PAGE) in 12\% gels and then transferred onto polyvinylidene fluoride (PVDF) membranes (EMD Millipore) using a Mini Trans-Blot electrophoretic transfer cell (Bio-Rad). Membranes were blocked in 5\% non-fat dry milk in PBS with Tween-20 (TPBS) and immunoblotting was performed with primary antibodies. HRP-conjugated secondary antibodies were used for detection. The immunoreactive bands were detected using Amersham ECL Plus Western Blotting Detection Reagents (GE Healthcare Life Sciences).

\section{Cloning, expression, and purification of recombinant proteins}

Cc was expressed in Escherichia coli BL21 (DE3) strain as previously described ${ }^{32}$. First, $25 \mathrm{~mL}$ Luria-Bertani (LB) medium, supplemented with $100 \mu \mathrm{g} / \mathrm{mL}$ ampicillin, were grown overnight at $37^{\circ} \mathrm{C}$ under agitation. Volume of 2.5 $\mathrm{mL}$ of pre-culture was used to inoculate $2.5 \mathrm{~L}$ of $\mathrm{LB}$ medium. The culture was incubated at $30^{\circ} \mathrm{C}$ under agitation for $24 \mathrm{~h}$. Then, cells were centrifuged at 6000 r.p.m. for $10 \mathrm{~min}$ in an Avanti J-25 centrifuge (Beckman Coulter). Following this, cells were re-suspended in $1.5 \mathrm{mM}$ borate buffer ( $\mathrm{pH} 8.5$ ), sonicated for $4 \mathrm{~min}$ and, finally, cellular debris was eliminated by centrifugation $(20,000 \mathrm{r}$. p.m. for $30 \mathrm{~min}$ ). For NMR measurements, ${ }^{15} \mathrm{~N}$-labeled $\mathrm{C} c$ was produced in minimal media with ${ }^{15} \mathrm{NH}_{4} \mathrm{Cl}$ as a unique nitrogen source. $\mathrm{Cc}$ purification was carried out by ionic chromatography with a carboxy-methylcellulose matrix. Fractions containing $\mathrm{C} c$ were concentrated in an Amicon ( $3 \mathrm{kDa}$ cutoff) until correct concentration was achieved.

$14-3-3 \varepsilon$ full length (14-3-3e FL) was cloned into the pET-28a vector under the T7 promotor using NdeI XhoI restriction sites and containing a N-terminal hexahistidine tag. For cloning, the following primers were used: 5'-AGCCATATGATGGATGATCGAGAGGAT-3' and 5'-GTGCTCGAGTCATTCCTGATTTTCGTC-3'. The 14-3-3e core was cloned with a Stop codon introduced after residue at position 233. The primers used for this mutagenesis were $5^{\prime}$-ACACTATGGACTTCATAGATGCAGGGTGACGGT-3' and 5'ACCGTCACCCTGCATCTATGAAGTCCATAGTGT3'. 14-3-3e FL D21K, K50E, S59E, E92K, D99K, and S187D mutants were obtained from Mutagenex Inc. (USA). The expression of 14-3-3e species were performed in the E. coli BL21 (DE3) strain. Cultures were grown in LB medium at $37^{\circ} \mathrm{C}$ until an O.D. $600 \mathrm{~nm}$ of $0.6-0.8$ was reached. Protein expression was induced by adding $1 \mathrm{mM}$ isopropyl- $\beta$-D-1-thiogalactopyranoside (IPTG) and cultures were grown for $24 \mathrm{~h}$ at $30^{\circ} \mathrm{C}$. The cells were collected by centrifugation and re-suspended in $20 \mathrm{mM}$ Tris$\mathrm{HCl}(\mathrm{pH}$ 8), $800 \mathrm{mM} \mathrm{NaCl}, 10 \mathrm{mM}$ imidazole. Cells were then broken by sonication and insoluble debris was removed by centrifugation, as indicated above. 14-3-3e species were purified by affinity chromatography, using a Ni Sepharose 6 Fast Flow (GE Healthcare) column and an imidazole gradient ranging from $10 \mathrm{mM}$ to $300 \mathrm{mM}$ imidazole. Protein containing fractions were concentrated in an Amicon (3 kDa cutoff) until the desired proper protein concentration was reached.

Proteins were dialyzed against $10 \mathrm{mM}$ sodium phosphate (pH 7.4) buffer for ITC measurements or $5 \mathrm{mM}$ sodium phosphate ( $\mathrm{pH}$ 6.3) buffer for NMR titrations. For caspase assays, protein was dialyzed against $20 \mathrm{mM}$ HEPES (pH 7.5) buffer.

14-3-3 $\varepsilon$ C-terminal peptide (14-3-3 $\left.\varepsilon_{234-255}\right)$ was purchased from Genosphere Biotechnologies (France).

Apaf- $1_{\triangle \mathrm{WD} 40}$ S268D and S268A mutants were obtained by mutagenic PCR from pET28a-Apaf- $1_{\triangle W D 40}$, using $5^{\prime}$ ACCAGAGACAAGGATGTTACAGATT- $3^{\prime}$ and $5^{\prime}$ AATCTGTAACATCCTTGTCTCTGGT-3' primers for the S268D mutant and 5'-ACCAGAGACAAGGCTGTTACAGATT-3' and 5'-AATCTGTAACAGCCTTGTCTCTGGT-3' primers for the S268A mutant. All Apaf- $1_{\triangle \mathrm{WD} 40}$ species were expressed as previously described with small modifications ${ }^{6}$. Protein expression was carried out in E. coli BL21 (DE3) at $20^{\circ} \mathrm{C}$ after induction with $1 \mathrm{mM}$ IPTG. Cells were broken by freeze/heat cycles and cellular debris was removed by centrifugation. The soluble fraction was purified by affinity chromatography (His.Trap column, GE Healthcare) followed by anionic-exchange (Hi.Trap column, GE Healthcare). Recombinant caspase-9 was expressed in $E$. coli BL21 (DE3) at $30^{\circ} \mathrm{C}$ and purified as indicated above for Apaf- $1_{\triangle W D 40}$. Protein quantification was assessed using the Bradford protein assay ${ }^{44}$.

\section{Caspase- 9 assays}

Caspase- 9 activity was measured as previously described $^{6}$. Recombinant Apaf- $1_{\triangle W D} 40$ species and 14-3-3 $\varepsilon$ FL were mixed in buffer (40 mM HEPES, pH 7.5, $20 \mathrm{mM} \mathrm{KCl}$, $1 \mathrm{mM}$ DTT) to a final concentration of $100 \mathrm{nM}$ and $1 \mu \mathrm{M}$, respectively. $\mathrm{C} c$ was added at different concentrations (ranging from 1 to $5 \mu \mathrm{M}$ ). After incubation for $15 \mathrm{~min}$ at room temperature, recombinant caspase- 9 was added to a final volume of $100 \mu \mathrm{L}$ and final concentration of $500 \mathrm{nM}$. After incubation for another $15 \mathrm{~min}$ at $37^{\circ} \mathrm{C}$, the caspase9 substrate LEHD peptide coupled to 7-amino-4trifluoromethylcoumarin (Ac-LEHD-AFC, TOCRIS) was added at a final concentration of $50 \mu \mathrm{M}$. The increase in fluorescence resulting from Ac-LEHD-AFC cleavage was determined in a Cary Eclipse (Varian) fluorescence spectrophotometer, using an excitation wavelength of $390 \mathrm{~nm}$ and an emission wavelength of $510 \mathrm{~nm}$. Each experimental data were the mean \pm SD value of at least three independent measurements. 


\section{Caspase- 3 assays}

In vitro caspase- 3 assays were performed as formerly described with slight changes ${ }^{45}$. Recombinant $\mathrm{C} c$ and 14$3-3 \varepsilon$ FL were mixed and incubated with $100 \mu \mathrm{g}$ of cytosolic HEK293T cells extracts for $60 \mathrm{~min}$ at $37^{\circ} \mathrm{C}$ in $10 \mathrm{mM}$ HEPES (pH 7.5) buffer, supplemented with $25 \mathrm{mM} \mathrm{KCl}$, $200 \mu \mathrm{M}$ DTT, $200 \mu \mathrm{M}$ dATP. After incubation, $10 \mathrm{mM}$ HEPES (pH 7.5), $50 \mathrm{mM} \mathrm{NaCl}, 40 \mathrm{mM} \beta$-glycerophosphate, $2 \mathrm{mM} \mathrm{MgCl}_{2}, 5 \mathrm{mM}$ EGTA, $0.1 \mathrm{mg} \mathrm{mL}^{-1}$ bovine serum albumin, and $0.1 \%(\mathrm{w} / \mathrm{v})$ 3-[3-cholamidopropyldimethylammonio]-1-propanesulfonate (CHAPS) buffer was added to a final volume of $200 \mu \mathrm{L}$, supplemented with $40 \mu \mathrm{M}$ of acetyl-DEVD-7-amino-4-methylcoumarin (AcDEVD-AMD, Enzo Life Sciences), a fluorescent substrate specific to caspase-3/7. The increase in fluorescence resulting from Ac-DEVD-AMC cleavage was determined in a Cary Eclipse (Varian) fluorescence spectrophotometer, using an excitation wavelength of $360 \mathrm{~nm}$ and an emission wavelength of $460 \mathrm{~nm}$. To compare the effect of $14-3-3 \varepsilon$ in different types of extracts, values for caspase-3 activity before adding recombinant $14-3-3 \varepsilon$ FL were normalized. Each experimental data were the mean \pm SD value of at least three independent measurements.

\section{ITC measurements}

All ITC experiments were performed using an AutoITC200 (MicroCal-Malvern Instruments, UK) at $25^{\circ} \mathrm{C}$ by titrating $14-3-3 \varepsilon$ species and $14-3-3 \varepsilon$ peptides with $\mathrm{Cc}$. The reference cell was filled with distilled water. The experiments consisted of $2-\mu \mathrm{L}$ injections of $300 \mu \mathrm{M} \mathrm{Cc}$ solution in $10 \mathrm{mM}$ sodium phosphate buffer ( $\mathrm{pH}$ 7.4) into the sample cell, initially containing $20 \mu \mathrm{M} 14-3-3 \varepsilon$ solution in the same buffer. All solutions were degassed before titrations were performed. Titrant was injected at appropriate time intervals to ensure the thermal power signal returned to the base line prior to the next injection. To achieve homogeneous mixing in the cell, the stirring speed was maintained constant at 750 r.p.m. The data, specifically the heat per injection normalized per mole of injectant vs. the molar ratio, were analyzed with Origin 7.0 (OriginLab Corp.). Calibration and performance tests of the calorimeter were carried out conducting $\mathrm{CaCl}_{2}$-EDTA titrations with solutions provided by the manufacturer. Control experiments ( $\mathrm{Cc}$ solution injected into buffer) were performed in order to assess potential unspecific heat effects.

\section{NMR spectroscopy}

NMR titration of reduced $C c$ with 14-3-3e FL was followed by $1 \mathrm{D}{ }^{1} \mathrm{H}$ and $2 \mathrm{D}\left[{ }^{1} \mathrm{H},{ }^{15} \mathrm{~N}\right]$ HSQC spectra on a Bruker Avance $700 \mathrm{MHz}$ at $25^{\circ} \mathrm{C} .50 \mu \mathrm{M}$ of reduced, ${ }^{15} \mathrm{~N}$ labelled $\mathrm{Cc}$ in $5 \mathrm{mM}$ sodium phosphate buffer ( $\mathrm{pH}$ 6.3) was titrated with unlabeled $14-3-3 \varepsilon$ in the same buffer. $0.1 \mathrm{M}$ sodium ascorbate and $10 \% \mathrm{D}_{2} \mathrm{O}$ were added in order to ensure the redox state remained constant and to adjust the lock signal, respectively. Water signal was suppressed according to the WATERGATE solvent suppression method ${ }^{46}$. The interaction of reduced $\mathrm{C} c$ with $14-3-3 \varepsilon_{234-255}$ was performed on a Bruker Avance 600 $\mathrm{MHz}$ in the same way as previously described for the titration. Data were processed using TopSpin NMR 2.0 software (Bruker). Line broadening and chemical-shift perturbation analysis were performed using Sparky 3 NMR Assignment Program (T.D. Goddard and D.G. Kneller, University of California - San Francisco, US). CSP titration curves were fitted using the one-site binding model.

To fluorinate the Lys244 side chain of $14-3-3 \varepsilon_{234-255}$, the bioconjugation reaction was performed with 5fluoroindole compound (Sigma) as previously described $^{47}$. $500 \mu \mathrm{M}$ acetylated $14-3-3 \varepsilon_{234-255}$ peptide was mixed with $2 \mathrm{mM}$ formaldehyde solution $(37 \% \mathrm{w} / \mathrm{w})$ and with $6 \mathrm{mM} \mathrm{5}$-fluoroindole for $24 \mathrm{~h}$ at $30^{\circ} \mathrm{C}$. Then, the sample was dialyzed against $10 \mathrm{mM}$ sodium phosphate (pH 6.3) buffer overnight. Proper folding of 14-3-3 $\varepsilon_{234-255}$ before and after the reaction was checked by $1 \mathrm{D}{ }^{1} \mathrm{H}$ spectra, and the interaction with $\mathrm{Cc}$ was followed recording $1 \mathrm{D}{ }^{19} \mathrm{~F}$ experiments at $25^{\circ} \mathrm{C}$ on a Bruker Avance $600 \mathrm{MHz}$.

\section{Analytical ultracentrifugation}

Sedimentation velocity experiments of $14-3-3 \varepsilon$ FL WT, D21K, K50E, S59E, E92K, D99K, and S187D were performed at $20^{\circ} \mathrm{C}$ in an Optima XL-A Analytical Ultracentrifuge (AU, Beckman Instruments) with an AN50-Ti rotor. These experiments were carried out at 45,000 r.p.m. with $400-\mu \mathrm{L}$ samples at $37 \mu \mathrm{M}$ in $5 \mathrm{mM}$ sodium phosphate buffer ( $\mathrm{pH}$ 6.3) loaded into double sector cells. Radial scans at $280 \mathrm{~nm}$ were taken every $10 \mathrm{~min}$ and the sedimentation coefficient distribution was calculated by least squares boundary modeling of the sedimentation velocity data using the programme SEDFIT. The experimental coefficients were converted to standard conditions $(\mathrm{s} 20, \mathrm{w})$.

\section{Molecular dynamic computations}

Molecular dynamics (MD) computations were carried out using the AMBER 12 package and the AMBER-2003 force field. For the 14-3-3 $\varepsilon_{234-255}$ peptide simulation, an unfolded and outspread conformation was used as initial coordinates. After $100 \mathrm{~ns}$ of simulation, the closest structure to the average from the last $40 \mathrm{~ns}$ of this computation was attached to the crystallographic structure of

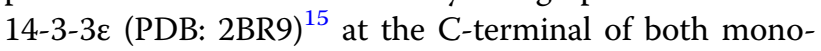
mers. The resulting structure was used as the initial coordinate for the full length protein in the MD computation. With the aim to promote the opening of 14-3-3e $\mathrm{FL}$ and to produce an open conformation, both consensus 
peptide ligands for 14-3-3 proteins included in the crystallographic structure were removed from the initial coordinates.

All simulations were carried out under periodic boundary conditions using an orthorhombic cell geometry (minimum distance between protein and cell faces was initially set to $10 \AA$ ) and PME electrostatics were set with the Ewald summation cutoff at $9 \AA$. Sodium counterions were added to neutralize the charges of the system. The structures were solvated with SPC water molecules. Afterwards, solvent and counter-ions were subjected to 2000 steps of energy minimization followed by $300 \mathrm{ps}$ NPT-MD computations using isotropic molecule position scaling and a pressure relaxation time of 1 ps at $298 \mathrm{~K}$. The density of the system reached a plateau during the first 150 ps. Then, the whole system was energy minimized and submitted to NVT MD computations at $298 \mathrm{~K}$. The SHAKE algorithm ${ }^{48}$ was used to constrain bonds involving hydrogen atoms. The PTRAJ module of AMBER was used for trajectory analysis.

\section{NMR-driven docking}

Restrained docking calculations were performed with the High Ambiguity Driven Docking approach (HADDOCK $)^{49-51}$, using the 3ZCF.pdb structure of $\mathrm{C} c$ and the resulting open model of $14-3-3 \varepsilon$ FL from MD calculations. Dielectric constant was set as distance-dependent. Scaling of ambiguous interaction restraints (AIRs) were fixed to 0.1 for rigid body and to 1.0 for both semi-flexible simulated annealing and final refinement. AIRs for the docking simulation were generated using standard criteria. Random exclusion of AIRs was not employed. Cc residues labeled as active were those showing ${ }^{1} \mathrm{H} \Delta \Delta v_{1 /}$ 2Binding $\geq 59.2 \mathrm{~Hz}$ and a solvent accessibility larger than $50 \%$ calculated with NACCESS. These active residues were Gln16, Gln42, Ser47, Ala50, Gly77, Lys79, Ile81, and Val83. Cc residues located at $<4 \AA$ from the active ones and showing high-solvent accessible surface $(>50 \%)$ were labeled as passive residues. $14-3-3 \varepsilon$ residues labeled as active were those whose mutation resulted in the impairment of one of the Cc-binding sites: Asp21, Lys50, Ser59, and Glu92. 14-3-3e residues located at $<4 \AA$ from the active ones and showing high-solvent accessible surface $(>50 \%)$ were labeled as passive residues. For each run, 10,000 rigid-body solutions were generated by energy minimization. The 1000 structures with the lowest AIRs energies were subjected to semi-flexible simulated annealing. The first 500 structures were submitted to a final refinement in explicit water. The 500 best structures were analyzed using standard criteria. AIR violations were calculated using as reference the solution with best HADDOCK score. Structures were represented using UCSF Chimera ${ }^{52}$.

\section{Dynamic light scattering measurements}

Dynamic light scattering experiments were performed to evaluate the dimerization state of 14-3-3ع FL WT and D21K, K50E, S59E, E92K, D99K, and S187D mutants, in 5 $\mathrm{mM}$ sodium phosphate at $\mathrm{pH}$ 6.3. DLS experiments on $0.1 \mathrm{mg} / \mathrm{mL}$ of each $14-3-3 \varepsilon$ species were conducted at $25^{\circ}$ $\mathrm{C}$ in a Zetasizer Nano ZS (Malvern Instruments).

\section{Statistical analysis}

Statistical analysis was performed using GraphPad Prism 7 Software and experimental data of at least three independent measurements. Statistical significance was estimated using Student's $t$-test. A $p$-value of $<0.0002$ was considered significant.

\section{Acknowledgements}

This work was supported by Spanish Ministry of Economy, Industry and Competitiveness (BFU2015-71017/BMC, SAF2015-64383-P and BFU201347064P/BMC MINECO/FEDER, EU), Ramon Areces Foundation (2015-2017), European Social Fund 2007-2013, Andalusian Government (BIO198 and BIO778), Cámara Foundation — University of Seville grant awarded to C.A.E.-R., BIP-cicCartuja and CITIUS platforms.

\section{Author details}

${ }^{1}$ Instituto de Investigaciones Químicas (IIQ) - Centro de Investigaciones Científicas Isla de la Cartuja (cicCartuja), Universidad de Sevilla - Consejo Superior de Investigaciones Científicas (CSIC), Sevilla, Spain. ${ }^{2}$ Institute of Biocomputation and Physics of Complex Systems (BIFI), Joint Unit IQFR-CSICBIFI, Universidad de Zaragoza, Zaragoza, Spain. ${ }^{3}$ Centro de Investigación Príncipe Felipe, Valencia, Spain. ${ }^{4}$ Centro Andaluz de Biología Molecular y Medicina Regenerativa-CABIMER, CSIC-Universidad de Sevilla-Universidad Pablo de Olavide, Sevilla, Spain

\section{Authors contributions}

C.A.E.-R., A.D.-Q., K.G.-A., I.D.-M., and M.A.D.I.R. designed the research; C.A.E.-R. and A.V.-C. performed and analysed ITC measurements; C.A.E.-R. and M.O. cloned, expressed and purified APAF-1 mutants. C.A.E.-R., K.G.-A., and A.L.-R. carried out cellular experiments. C.A.E.-R., S.G.-C., A.D.-Q., and I.D.-M. performed and analysed NMR data, MD and docking calculations; C.A.E.-R., A.D.-Q., I.D.-M. and M.A.D.I.R. wrote the paper.

\section{Conflict of interest}

The authors declare that they have no conflict of interest.

\section{Publisher's note}

Springer Nature remains neutral with regard to jurisdictional claims in published maps and institutional affiliations.

Supplementary Information accompanies this paper at https://doi.org/ 10.1038/s41419-018-0408-1.

Received: 12 September 2017 Revised: 14 February 2018 Accepted: 16 February 2018

Published online: 06 March 2018

\footnotetext{
References

1. Jacobson, M. D., Weil, M. \& Raff, M. C. Programmed cell death in animal development. Cell 88, 347-354 (1997).

2. Krammer, P. H. CD95's deadly mission in the immune system. Nature 407, 789-795 (2000).
} 
3. Suen, D. F., Norris, K. L. \& Youle, R. J. Mitochondrial dynamics and apoptosis. Genes Dev. 22, 1577-1590 (2008).

4. Yang, J. et al. Prevention of apoptosis by Bcl-2: release of cytochrome $c$ from mitochondria blocked. Science 275, 1129-1132 (1997).

5. Díaz-Moreno, I., García-Heredia, J. M., Díaz-Quintana, A. \& De la Rosa, M. A. Cytochrome c signalosome in mitochondria. Eur. Biophys. J. 40, 1301-1315 (2011).

6. Riedl, S. J., Li, W., Chao, Y., Schwarzenbacher, R. \& Shi, Y. Structure of the apoptotic protease-activating factor 1 bound to ADP. Nature 434, 926-933 (2005).

7. Li, P. et al. Cytochrome $c$ and dATP-dependent formation of Apaf-1/Caspase-9 complex initiates an apoptotic protease cascade. Cell 91, 479-489 (1997).

8. Zou, H., Li, Y., Liu, X. \& Wang, X. An Apaf-1-cytochrome c multimeric complex is a functional apoptosome that activates procaspase-9. J. Biol. Chem. 274, 11549-11556 (1999).

9. Martínez-Fábregas, J. et al. New Arabidopsis thaliana cytochrome c partners: a look into the elusive role of cytochrome $\mathrm{c}$ in programmed cell death in plants. Mol. Cell. Proteom. 12, 3666-3676 (2013).

10. Martínez-Fábregas, J. et al. Structural and functional analysis of novel human cytochrome c targets in apoptosis. Mol. Cell. Proteom. 13, 1439-1456 (2014).

11. González-Arzola, K. et al. Structural basis for inhibition of the histone chaperone activity of SET/TAF-I $\beta$ by cytochrome c. Proc. Natl Acad. Sci. USA 112 , 9908-9913 (2015).

12. Martínez-Fabregas, J., Díaz-Moreno, I., González-Arzola, K., Díaz-Quintana, A. \& De la Rosa, M. A. A common signalosome for programmed cell death in humans and plants. Cell Death Dis. 5, e1314 (2014).

13. Moore, B. \& Perez, V. Physiological and biochemical aspects of nervous integration. In: F. Carlson (ed). Specific acidic proteins of the nervous system (pp. 343-359. Prentice-Hall, Englewood Cliffs, NJ, 1967).

14. Fu, H., Subramanian, R. R. \& Masters, S. C. 14-3-3 proteins: structure, function, and regulation. Annu. Rev. Pharmacol. Toxicol. 40, 617-647 (2000).

15. Yang, $X$. et al. Structural basis for protein-protein interactions in the 14-3-3 protein family. Proc. Natl Acad. Sci. USA 103, 17237-17242 (2006).

16. Petosa, C. et al. 14-3-3 $\zeta$ binds a phosphorylated raf peptide and an unphosphorylated peptide via its conserved amphipathic groove. J. Biol. Chem. 273, 16305-16310 (1998).

17. Masters, S. C., Pederson, K. J., Zhang, L., Barbieri, J. T. \& Fu, H. Interaction of 14-33 with a nonphosphorylated protein ligand, exoenzyme $S$ of Pseudomonas aeruginosa. Biochemistry 38, 5216-5221 (1999).

18. Aachmann, F. L., Fomenko, D. E., Soragni, A., Gladyshev, V. N. \& Dikiy, A Solution structure of selenoprotein W and NMR analysis of its interaction with 14-3-3 proteins. J. Biol. Chem. 282, 37036-37044 (2007)

19. Aitken, A., Ellis, C. A., Harris, A., Sellers, L. A. \& Toker, A. Kinase and neurotransmitters. Nature 344, 594-594 (1990).

20. Toker, A., Ellis, C. A., Sellers, L. A. SpringerAmpamp; Aitken, A. Protein kinase C inhibitor proteins. Eur. J. Biochem. 191, $421-429$ (1990).

21. Aitken, A., Howell, S., Jones, D., Madrazo, J. \& Patel, Y. 14-3-3 and are the phosphorylated forms of raf-activating 14-3-3 and in vivo stoichiometric phosphorylation in brain at a Ser-Pro-Glu-Lys motif. J. Biol. Chem. 270, 5706-5709 (1996).

22. Tsuruta, F. et al. JNK promotes Bax translocation to mitochondria through phosphorylation of 14-3-3 proteins. EMBO J. 23, 1889-1899 (2004).

23. Darling, D. L., Yingling, J. \& Wynshaw-Boris, A. Role of $14-3-3$ proteins in eukaryotic signaling and development. Curr. Top Dev. Biol. 68, 281-315 (2005).

24. Paul, G. \& van Heusden, H. 14-3-3 proteins: regulators of numerous eukaryotic proteins. IUBMB Life. 57, 623-629 (2005)

25. Kim, J. et al. Rsk-mediated phosphorylation and 14-3-3ع binding of Apaf-1 suppresses cytochrome c-induced apoptosis. EMBO J. 31, 1279-1292 (2012).

26. Allan, L. Y. et al. Inhibition of caspase- 9 through phosphorylation at Thr 125 by ERK MAPK. Nat. Cell. Biol. 5, 647-654 (2003).

27. Palacios-Rodriguez, Y. et al. Polypeptide modulators of caspase recruitment domain (CARD)-CARD-mediated protein-protein interactions. J. Biol. Chem. 286, 44457-44466 (2011).

28. Ma, Y. Sphingosine activates protein kinase A type $\|$ by a novel CAMPindependent mechanism. J. Biol. Chem. 280, 26011-26017 (2005).

29. Gu, Y. M. et al. Protein kinase A phosphorylates and regulates dimerization of 14-3-3ろ. FEBS Lett. 580, 305-310 (2006).
30. Kim, J. S., Diebold, B. A., Babior, B. M., Knaus, U. G. \& Bokoch, G. M. Regulation of Nox1 activity via protein kinase A-mediated phosphorylation of NoxA1 and 14-3-3 binding. J. Biol. Chem. 282, 34787-34800 (2007).

31. Moreno-Beltrán, B. et al. Cytochrome $c_{1}$ exhibits two binding sites for cytochrome $\mathrm{c}$ in plants. Biochim. Biophys. Acta 1837, 1717-1729 (2014).

32. Moreno-Beltrán, B. et al. Respiratory complexes III and IV can each bind two molecules of cytochrome $c$ at low ionic strength. FEBS Lett. 589, 476-483 (2015).

33. Sakamoto, K. et al. NMR basis for interprotein electron transfer gating between cytochrome $c$ and cytochrome c oxidase. Proc. Natl Acad. Sci. USA 108, 12271-12276 (2011).

34. Díaz-Moreno, I., Díaz-Quintana, A., Ubbink, M. \& De la Rosa, M. A. Different modes of interaction in cyanobacterial complexes of plastocyanin and cytochrome. F. Biochem. 44, 3176-3183 (2005).

35. Ferrell, J. E. \& Machleder, E. M. The biochemical basis of an all-or-none cell fate switch in Xenopus oocytes. Science 280, 895-898 (1998).

36. Díaz-Moreno, I., García-Heredia, J. M., Díaz-Quintana, A., Teixeira, M. \& De la Rosa, M. A. Nitration of tyrosines 46 and 48 induces the specific degradation of cytochrome $c$ upon change of the heme iron state to high-spin. Biochim. Biophys. Acta 1807, 1616-1623 (2011).

37. García-Heredia, J. M. et al. Specific nitration of tyrosines 46 and 48 makes cytochrome $c$ assemble a non-functional apoptosome. FEBS Lett. 586, 154-158 (2012).

38. Guerra-Castellano, A. et al. Mimicking tyrosine phosphorylation in human cytochrome $c$ by the evolved tRNA synthetase technique. Chemistry 21, 15004-15012 (2015).

39. Guerra-Castellano, A., Díaz-Moreno, l., Velázquez-Campoy, A., De la Rosa, M. A. \& Díaz-Quintana, A. Structural and functional characterization of phosphomimetic mutants of cytochrome $c$ at threonine 28 and serine 47. Biochim. Biophys. Acta 1857, 387-395 (2016).

40. Moreno-Beltrán, B. et al. Structural basis of mitochondrial dysfunction in response to cytochrome c phosphorylation at tyrosine 48. Proc. Natl Acad. Sci. USA 114, 3041-3050 (2017).

41. González-Arzola, K. et al. Histone chaperone activity of Arabidopsis thaliana NRP1 is blocked by cytochrome c. Nucleic Acids Res. 45, 2150-2165 (2017).

42. Kingston, R. E., Chen, C. A. \& Rose, J. K. Calcium phosphate transfection. Curr. Protoc. Mol. Biol. https://doi.org/10.1002/0471142727.mb0901s63. (John Wiley \& Sons, Inc., 2003)

43. Pecina, P. et al. Phosphomimetic substitution of cytochrome $c$ tyrosine 48 decreases respiration and binding to cardiolipin and abolishes ability to trigger downstream caspase activation. Biochemistry 49, 6705-6714 (2010).

44. Bradford, M. M. A rapid and sensitive method for the quantitation of microgram quantities of protein utilizing the principle of protein-dye binding. Anal. Biochem. 72, 248-254 (1976).

45. Slee, E. A. et al. Ordering the Cytochrome $c$-initiated caspase cascade: hierarchical activation of caspases-2, -3, -6, -7, -8, and -10 in a caspase9-dependent manner. J. Cell Biol. 144, 281-292 (1999).

46. Piotto, M., Saudek, V. \& Sklenár, V. Gradient-tailored excitation for singlequantum NMR spectroscopy of aqueous solutions. J. Biomol. NMR 2, 661-665 (1992).

47. Larda, S. T., Pichugin, D. \& Prosser, R. S. Site-specific labeling of protein lysine residues and $\mathrm{N}$-terminal amino groups with indoles and indole-derivatives. Bioconjug. Chem. 26, 2376-2383 (2015).

48. Ryckaert, J.-P., Ciccotti, G. \& Berendsen, H. J. C. Numerical integration of the cartesian equations of motion of a system with constraints: molecular dynamics of n-alkanes. J. Comput. Phys. 23, 327-341 (1977).

49. Dominguez, C., Boelens, R. \& Bonvin, A. M. J. J. HADDOCK: a protein-protein docking approach based on biochemical or biophysical information. J. Am. Chem. Soc. 125, 1731-1737 (2003).

50. van Dijk, A. D. J., Boelens, R. \& Bonvin, A. M. J. J. Data-driven docking for the study of biomolecular complexes. FEBS. J. 272, 293-312 (2005).

51. de Vries, S. J. et al. HADDOCK versus HADDOCK: new features and performance of HADDOCK2.0 on the CAPRI targets. Proteins 69, 726-733 (2007).

52. Pettersen, E. F. et al. UCSF Chimera-A visualization system for exploratory research and analysis. J. Comput. Chem. 25, 1605-1612 (2004). 